\title{
Effect of zinc supplementation on lipid peroxidation and lactate levels in rats with diabetes induced by streptozotocin and subjected to acute swimming exercise
}

\author{
Bicer $\mathrm{M}^{1}$, Gunay $\mathrm{M}^{2}$, Baltaci $\mathrm{AK}^{3}$, Uney $\mathrm{K}^{4}$, Mogulkoc $\mathrm{R}^{3}$, Akil $\mathrm{M}^{5}$ \\ Selcuk University School of Physical Education and Sports, Konya, Turkey. murselbicer@yahoo.com
}

\begin{abstract}
Objective: The present study aims to explore the effect of zinc supplementation on lipid peroxidation and lactate levels in rats having diabetes induced by streptozotocin and subjected to acute swimming exercise Method: A total of 80 adult male rats of Sprague-Dawley type were equally allocated to 8 groups: Group 1, general control. Group 2, zinc-supplemented group. Group 3, zinc-supplemented, diabetic group. Group 4, swimming control group. Group 5, zinc-supplemented swimming group. Group 6, zinc-supplemented diabetic swimming group. Group 7, diabetic swimming group. Group 8, diabetic group. At the end of the 4-week study, blood samples were collected to determine MDA, GSH, GPx, SOD, lactate and zinc levels.

Results: The highest MDA values were found in group 7 and 8 ( $p<0.001)$. GSH values in groups 5 and 6 were higher $(p<0.001)$. The highest GPx values were established in groups 2,5 and $6(p<0.001)$. SOD values were the highest in groups 5 and $6(p<0.001)$ and lowest in groups 2,3 and $8(p<0.001)$. The highest plasma lactate levels were found in group $7(p<0.001)$. The highest zinc levels were obtained in groups 1,2 and $5(p<0.001)$, and the lowest zinc levels were found in groups 7 and $8(p<0.001)$.

Conclusion: Results of the study reveal that zinc supplementation prevents the increase in free radical formation, suppression of antioxidant activity and muscle exhaustion, all of which result from diabetes and acute exercise. Zinc supplementation may contribute to health and performance in diabetes and acute exercise (Tab. 2, Fig. 1 Ref. 47). Full Text in PDF www.elis.sk.

Key words: zinc, diabetes, exercise, free radicals, rat.
\end{abstract}

Diabetes Mellitus is a chronic metabolic disease, characterized by hyperglycemia, as well as insufficient or ineffective secretion of endogenous insulin (1). Type 1 diabetes arises from severe damage to pancreatic cells and frequently results in insulin dependence. The major mechanism believed to contribute to beta cell damage is oxidative stress. Hydroxyl free radicals stimulated by hyperglycemia and various free radicals including reactive oxygen species (ROS) are responsible for the destruction of pancreas beta cells as a result of oxidative stress. Therefore, studies about the treatment and prevention of diabetes have dealt with not only conditions involving oxidative stress, but also prevention with antioxidants (2).

The relationship between zinc, and pancreas and insulin has been known for a long time. Zinc is found in beta and alpha cells of the pancreas. It has been argued that zinc plays a role in the production, storage and secretion of insulin in beta cells in particular (3).

${ }^{1}$ Selcuk University School of Physical Education and Sports, Konya, Turkey, ${ }^{2}$ Gazi University School of Physical Education and Sports, Ankara, Turkey, ${ }^{3}$ Selcuk University, Selcuklu Medical School, Department of Physiology, Konya, Turkey, ${ }^{4}$ Selcuk University Faculty of Veterinary Medicine, Department of Pharmacology and Toxicology, Konya, Turkey, and ${ }^{5}$ Karabuk University Hasan Dogan School of Physical Education and Sports, Karabuk, Turkey

Address for correspondence: M. Bicer, D, Selcuk University, High School of Physical Education and Sport, Konya, Turkey.
Zinc, which plays a critical role in many events, including endocrinology and the immune system in particular, is well-tolerated at the recommended doses (4). Among the main functions of zinc is its antioxidant role and involvement in the antioxidant defense system. Zinc exerts its antioxidant effect through two mechanisms. Firstly, redox-stable zinc substitutes for redox-reactive materials like iron and copper in critical cellular and extracellular regions. Secondly, it induces the synthesis of metallothioneins, which are sulphydryl-rich proteins protective against free radicals $(4,5)$.

Regular exercise and physical activity are also accepted to have a protective effect against cardiovascular diseases and mortality (6). Accordingly, exercise is strongly recommended to diabetic patients (7). However, it was also reported that muscle exercises led to an increase in the production of radicals and other reactive oxygen species (8).

Despite all these, there is not enough information pertinent to pros and cons of acute and chronic exercise associated with oxidative stress in groups with enhanced susceptibility to oxidative stress, like diabetic patients (9).

The present study aimed to explore the effect of zinc supplementation on lipid peroxidation, antioxidant capacity and lactate levels in rats which had diabetes induced by streptozotocin and were subjected to acute swimming exercise. 


\section{Materials and methods}

\section{Animal material and groups}

The present study was conducted at the Experimental Animals Unit of Selcuk University, Faculty of Veterinary Medicine (KonyaTurkey), using 80 Spraque-Dawley type adult (4-6 month-old and weighing 200-250 g) male rats obtained from the Experimental Medicine Application and Research Centre of Mediterranean University (Antalya-Turkey). The study protocol was approved by the local ethics committee.

The animals used in the study were equally divided into 8 groups:

Group 1, $(\mathrm{n}=10)$ General Control Group: The group which was fed on normal diet and was not subjected to any procedure.

Group 2, (n=10) Zinc-Supplemented Control Group: The group which was fed on normal diet, and additionally supplemented with $6 \mathrm{mg} / \mathrm{kg} /$ day intraperitoneal zinc sulfate for 4 weeks.

Group 3, (n=10) Zinc-Supplemented Diabetic Control Group: The group which was supplemented with $6 \mathrm{mg} / \mathrm{kg} /$ day intraperitoneal zinc sulfate for 4 weeks after the inducement of diabetes by subcutaneous " $40 \mathrm{mg} / \mathrm{kg}$ ” streptozotocin (STZ) administration.

Group 4, (n=10) Swimming Control Group: The group which was fed on normal diet and subjected to acute swimming exercise for 30 minutes.

Group 5, (n=10) Zinc-Supplemented Swimming Group: The group which was fed on normal diet, supplemented with $6 \mathrm{mg} / \mathrm{kg}$ / day intraperitoneal zinc sulfate for 4 weeks and subjected to acute swimming exercise for 30 minutes.

Group 6, $(\mathrm{n}=10)$ Zinc-Supplemented Diabetic Swimming Group: The group in which diabetes was induced with subcutaneous "40 mg/kg" streptozotocin (STZ), and which, thereafter, was supplemented with $6 \mathrm{mg} / \mathrm{kg} /$ day intraperitoneal zinc sulfate for 4 weeks and subjected to acute swimming exercise for 30 minutes.

Group 7, $(\mathrm{n}=10)$ Diabetic Swimming Group: The group in which diabetes was induced with subcutaneous " $40 \mathrm{mg} / \mathrm{kg}$ ” streptozotocin (STZ), and which was subjected to acute swimming exercise for 30 minutes.

Group 8, $(\mathrm{n}=10)$ Diabetes Group: The group in which diabetes was induced with subcutaneous " $40 \mathrm{mg} / \mathrm{kg}$ " streptozotocin (STZ).

\section{Experimental Animals}

The experimental animals were fed in special steel cages, which were washed and cleaned daily. The feed was given in special steel feeding bowls and water (usual tap water) in glass feeding bottles. The animals were kept in an environment which had 12 hour dark/12 hour light cycles and standard room temperature $\left(21 \pm 1^{\circ} \mathrm{C}\right)$. All injections were given between 09.00 and 10.00 in the morning. After diabetes was induced, the animals were immediately put on i.p. zinc sulphate for 4 weeks. Following the end of 4-week procedures, blood samples to be used in the analyses were collected from all animals by decapitation at 09.00-10.00 a.m. The collected samples were kept at $-80{ }^{\circ} \mathrm{C}$ until the analysis time.

\section{Experimental procedures}

\section{Inducement of diabetes in experimental animals}

In order to induce diabetes, 40 rats were set aside as diabetic groups. The rats were injected with $40 \mathrm{mg} / \mathrm{kg}$ intraperitoneal streptozotocin (STZ) "Sigma, S-0130". The injections were repeated at the same dose after 24 hours. Six days after the last injection, blood glucose levels of the animals were measured from the tail vein, using a diagnostic glucose kit. Animals whose blood glucose was at or above $300 \mathrm{mg} / \mathrm{dlt}$ were considered diabetic (10). As there was no mortality in diabetes-induced animals, group numbers remained unchanged.

\section{Zinc sulfate supplementation}

After being dissolved in distilled water, in $0.5 \mathrm{ml}$ serum physiologic $6 \mathrm{mg} / \mathrm{kg} /$ day zinc sulfate was supplemented by intraperitoneal injection. Zinc sulfate supplementation was repeated for 4 weeks at around the same hour (09.00 a.m.).

\section{Swimming exercise}

The exercise was performed in a heat-resistant, glass swimming pool which was $50 \mathrm{~cm}$ in depth and width, and had a thermostat to keep the temperature fixed at $37{ }^{\circ} \mathrm{C}$. Exercises were performed only once for 30 minutes, 24 hours after the end of zinc sulphate supplementations. After the experimental animals were made to swim in pairs, their blood samples were collected by decapitation for analysis. From the blood sample of each animal, a volume of $2 \mathrm{ml}$ was put into two tubes each, one with EDTA. The samples were centrifuged at 3000 rotation (rpm) for 10 minutes to separate plasma and serum.

\section{Biochemical analyses}

Plasma MDA (malondialdehyde) measurements

Although MDA can be analyzed in both plasma and serum, we carried out the MDA analysis in the plasma, as described by Draper and Hadley (11).

Plasma MDA levels were determined using thiobarbituric acid method. Absorbance of thiobarbituric acid-MDA complex was measured at $532 \mathrm{~nm}$ (Shimadzu UV-1061, Japan) (11). Plasma MDA levels were determined as $\mathrm{nmol} / \mathrm{ml}$.

\section{Erythrocyte GSH (reduced glutathione) quantifications}

Erythrocytes are the most glutathione-rich tissue. Therefore, measurement of GSH in the erythrocytes will produce more reliable results than plasma GSH measurement (12). That's why GSH measurement was conducted in erythrocytes in our study. Erythrocytes were washed three in 1/5 serum physiologic. Erythrocyte GSH levels was determined according to Ellmann method (12). Their absorbances were read against distilled water at $412 \mathrm{~nm}$. The values were presented as $\mathrm{mg} / \mathrm{dl}$.

Serum glutathione peroxidase (GPx) analyses

GPx analyses were conducted according to ELISA colorimetric method using a Cayman trademark (Catalogue No: 703102) commercial kit. The detection range of the kit was between 50 and $344 \mathrm{nmol} / \mathrm{min} / \mathrm{ml}$. The absorbance of this reaction was measured at $340 \mathrm{~nm}$. Results were expressed as $\mathrm{nmol} / \mathrm{ml}$.

Serum superoxide dismutase (SOD) analyses

SOD analyses were carried out in accordance with ELISA colorimetric method using a Cayman trademark (Catalogue No: 
706002) commercial kit. The detection range of the kit was between 0.025 and 0.25 units $/ \mathrm{ml}$. The results were determined as $\mathrm{U} / \mathrm{ml}$. Plasma lactate measurements

Plasma lactate levels were studied in TECHNICON RA-XT trademark autoanalyzer (Spinreact-make "Catalogue No: 703102" $5 \mathrm{X} 10 \mathrm{ml}$ packaged kit). The detection range of the kit was between 0.39 and $150 \mathrm{mg} / \mathrm{dl}$. Plasma lactate levels were measured (by being read at $550 \mathrm{~nm}$ ) as $\mathrm{mg} / \mathrm{dl}$.

Serum zinc quantifications

Analyses of serum zinc levels were carried out using the inductively coupled plasma emission spectrophotometry (ICP-AES; Varian Australia Pty LTD, Australia) atomic emission spectrophotometer found at the Department of Soil Science of Selcuk University, Faculty of Agriculture. Zinc levels were presented as $\mu \mathrm{g} / \mathrm{dl}$. Statistical evaluations

The data were analyzed using SPSS 10.3 software package. Statistical evaluation of the results was conducted using computer package software. Arithmetic means and standard errors of all other data were calculated. Variance analysis was used to determine differences. The Least Significant Difference Test ("LSD") was employed to determine the level of significance of the data which were found different in the variance analysis and to compare group means Differences for which $\mathrm{p}<0.05$ were accepted significant. The results were expressed as \pm standard deviation.

\section{Results and discussion}

The highest plasma MDA values were obtained in groups 7 (diabetic swimming group) and 8 (diabetes group). MDA values in groups 3 (zinc-supplemented diabetic control), 4 (swimming control) and 6 (zinc-supplemented diabetic swimming) were lower than those in groups 7 (diabetic swimming group) and 8 (diabetes group) ( $\mathrm{p}<0.001)$. Groups 1 (general control), 2 (zinc-supplemented control) and 5 (zinc-supplemented swimming group) had the lowest MDA values $(\mathrm{p}<0.001)$ (Tab. 1). A comparison of GSH values between groups revealed that groups 5 (zinc-supplemented swimming group) and 6 (zinc-supplemented diabetic swimming) had the highest and groups 1 (general control), 7 (diabetic swimming) and 8 (diabetes) had the lowest GSH levels $(\mathrm{p}<0.001)$ (Tab. 1).

The highest GPx values were found in groups 2 (zinc-supplemented control), 5 (zinc-supplemented swimming) and 6 (zinc-

Tab. 1. Plasma MDA (nmol/ml) and erythrocyte GSH (mg/dl) levels of study groups.

\begin{tabular}{lcc}
\hline Groups $(\mathrm{n}=10)$ & MDA & GSH \\
\hline 1 General Control & $1.48 \pm 0.37 \mathrm{D}$ & $28.72 \pm 6.41 \mathrm{D}$ \\
2 Zinc-Supplemented Control & $0.97 \pm 0.19 \mathrm{D}$ & $40.17 \pm 4.19 \mathrm{C}$ \\
3 Zinc-Supplemented Diabetic Control & $2.52 \pm 0.68 \mathrm{C}$ & $44.86 \pm 4.26 \mathrm{C}$ \\
4 Swimming Control & $2.46 \pm 0.56 \mathrm{C}$ & $39.74 \pm 3.64 \mathrm{C}$ \\
5 Zinc-Supplemented Swimming & $0.66 \pm 0.09 \mathrm{D}$ & $63.58 \pm 2.18 \mathrm{~A}$ \\
6 Zinc-Supplemented Diabetic Swimming & $2.46 \pm 0.27 \mathrm{C}$ & $52.43 \pm 1.35 \mathrm{~B}$ \\
7 Diabetic Swimming & $5.39 \pm 0.91 \mathrm{~A}$ & $30.05 \pm 3.63 \mathrm{D}$ \\
8 Diabetes & $3.87 \pm 0.73 \mathrm{~B}$ & $27.25 \pm 2.49 \mathrm{D}$ \\
\hline
\end{tabular}

*Means with different superscripted letters in the same column indicate statistical significance ( $p<0.001)$, MDA: $A>B C D, B>C D, C>D, G S H: A>B C D, B>C D, C>D$
Tab. 2. Serum GPx (nmol/ml)and SOD $(\mathrm{U} / \mathrm{ml})$ levels of study groups.

\begin{tabular}{lcc}
\hline Groups $(\mathrm{n}=10)$ & GPx & SOD \\
\hline 1 General Control & $118.45 \pm 17.92 \mathrm{D}$ & $0.45 \pm 0.03 \mathrm{D}$ \\
2 Zinc-Supplemented Control & $194.22 \pm 51.53 \mathrm{~B}$ & $0.66 \pm 0.05 \mathrm{~B}$ \\
3 Zinc-Supplemented Diabetic Control & $142.45 \pm 15.92 \mathrm{C}$ & $0.57 \pm 0.08 \mathrm{C}$ \\
4 Swimming Control & $123.69 \pm 20.29 \mathrm{D}$ & $0.56 \pm 0.02 \mathrm{C}$ \\
5 Zinc-Supplemented Swimming & $232.72 \pm 37.45 \mathrm{~A}$ & $0.93 \pm 0.05 \mathrm{~A}$ \\
6 Zinc-Supplemented Diabetic Swimming & $177.19 \pm 19.09 \mathrm{~B}$ & $0.70 \pm 0.07 \mathrm{~B}$ \\
7 Diabetic Swimming & $117.60 \pm 19.40 \mathrm{D}$ & $0.47 \pm 0.04 \mathrm{D}$ \\
8 Diabetes & $131.20 \pm 20.07 \mathrm{D}$ & $0.50 \pm 0.01 \mathrm{D}$ \\
\hline
\end{tabular}

*Means with different superscripted letters in the same column indicate statistical significance $(\mathrm{p}<0.001)$, GPx: $A>B C D, B>C D, C>D$, SOD: $\mathrm{A}>\mathrm{BCD}, \mathrm{B}>\mathrm{CD}, \mathrm{C}>\mathrm{D}$

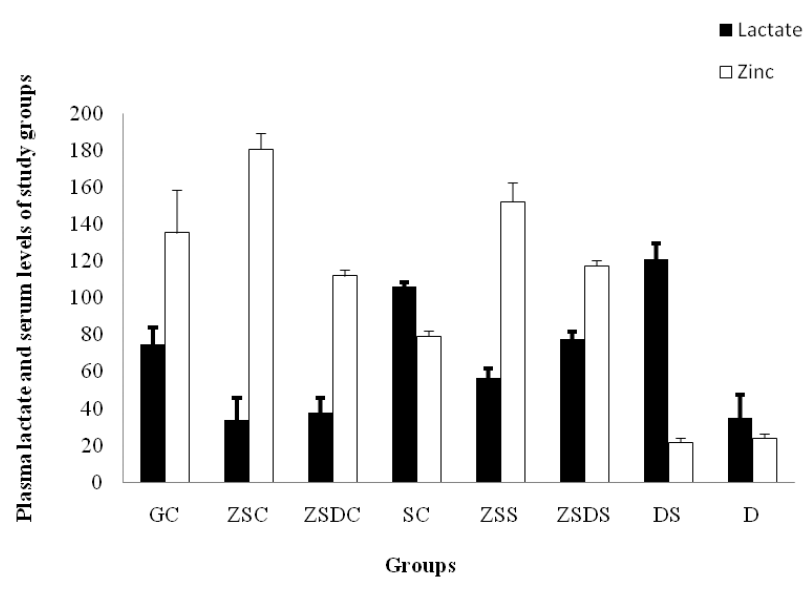

Fig. 1. Plasma lactate and serum zinc levels of subjects. GC -- general control; ZSC --zinc-supplemented control; ZSDC -- zinc-supplemented diabetic control; SC -- swimming control; ZSS -- zinc-supplemented swimming; ZSDS -- zinc-supplemented diabetic swimming; DS -- diabetic swimming; $\mathrm{D}$ - diabetes.

supplemented diabetic swimming) $(\mathrm{p}<0.001)$. Groups 1 (general control), 4 (swimming control), 7 (diabetic swimming) and 8 (diabetic control) had the lowest GPx levels $(\mathrm{p}<0.001)$ (Tab. 2). SOD values of the study groups are presented in Table 3. Accordingly, the highest SOD values were obtained in groups 5 (zinc-supplemented swimming) and 6 (zinc-supplemented diabetic swimming) $(\mathrm{p}<0.001)$. Groups 1,7 and 8 were observed to have the lowest SOD values $(\mathrm{p}<0.001)$ (Tab. 2).

When the groups were compared in terms of their plasma lactate values, it was established that groups 4 (swimming control) and 7 (diabetic swimming) had significantly higher lactate values than all other groups $(\mathrm{p}<0.001)$. The lowest plasma lactate levels were found in groups 2 (zinc-supplemented control), 3 (zincsupplemented diabetic control) and 8 (diabetes group) $(\mathrm{p}<0.001$, Graph 1). Among the study groups, groups 1 (general control), 2 (zinc-supplemented control) and 5 (zinc-supplemented swimming) were seen to have the highest serum zinc values, while groups 7 (diabetic swimming) and 8 (diabetes) had the lowest serum zinc levels ( $\mathrm{p}<0.001$, Graph 1).

It is known that lipid peroxidation significantly increases in diabetic conditions $(13,14)$. In this respect, high plasma MDA values we obtained in group 7 may be seen as an expected result 
and are in harmony with the results of the abovementioned researchers. However, group 7 is the group which was subjected to acute swimming exercise together with the inducement of diabetes. Despite conflicting data, it is generally accepted that physical exercise increases free radical formation (15). Oxygen intake of the whole body during heavy exercise may get as high as 20 times of the resting level. However, there may a 200 -fold increase in the oxygen consumption by active muscle fibers (16). The resulting mitochondrial metabolic leaks lead to a rise in free radical production (17). High MDA values we found in group 7 in which diabetes was induced and which was subjected to acute swimming exercise are consistent with studies reported on this topic. In addition to being a chronic metabolic disorder, diabetes is also a condition of enhanced oxidative stress (18). Elevated free radicals in diabetes interact with lipids, proteins and nucleic acids, and lead to a loss of membrane integrity, structural and functional modifications in proteins and genetic mutations (18). High MDA levels we obtained in group 8 may be accepted as an expected result and the result we obtained lends support to the reports of researchers who argued that lipid peroxidation increased in diabetes $(13,14,19)$. It is very remarkable that group 3 (zinc-supplemented diabetic control) and group 6 (zinc-supplemented diabetic swimming) were found to have lower plasma MDA values than groups 7 and 8 . The fact that low MDA values were obtained in groups 3 and 4 , relative to groups 7 and 8 , indicates that zinc supplementation curtailed the lipid peroxidation that was elevated both by diabetes and exercise. There is a growing body of evidence placing an emphasis on the antioxidant character of zinc (20). Previous studies have demonstrated that dietary zinc deficiency increases lipid peroxidation in rats $(21,22)$. Additionally, it was also shown that elevated MDA levels in the tissues of zinc-deficient rats were inhibited by zinc supplementation (23). Results of the researchers cited above indicate that zinc is significantly associated with the antioxidant system. A similar study with which we can compare the low MDA values we obtained in group 3 of our study is the one where Duzguner and Kaya (24) reported that the increased lipid peroxidation in diabetic rabbits subsided with zinc supplementation. The results of this study are important in that they support the low MDA values we found in group 3 (24). The low MDA values we established in zinc-supplemented groups (groups 2 and 5) are in harmony with the results of studies which argue that zinc supplementation increases antioxidant capacity and prevents lipid peroxidation $(20,25)$.

Many studies seem to concentrate on the relation between antioxidant activity and zinc in exercise $(24,26)$. The report to the effect that zinc-deficient diet inhibited the antioxidant system in trained rats subjected to swimming exercise (27) or the demonstration that zinc supplementation elevated the GSH levels which were inhibited in rats subjected to intense swimming exercise (28) lends support to increased GSH levels we found in group 5. GSH values in the zinc-supplemented diabetic swimming group (group 6) were lower than the levels in group 5 but higher than those in all the remaining groups. It can be argued that this finding we had in group 6 has resulted from zinc supplementation. That is because we found the lowest GSH values in the present study in the dia- betic swimming group (group 7) and non-exercised diabetes group (group 8), which were not supplemented with zinc.

Many researchers reported that antioxidant activity was inhibited in diabetes $(29,30)$. The reduced GSH values we found in groups 7 and 8 constitute an expected complication of diabetes. What needs to be underlined here is that zinc supplementation elevated the GSH values which were reduced by diabetes in the diabetic swimming group (group 6).

In our study, we obtained a similar finding in the zinc-supplemented diabetic control group (group 3), which had higher GSH levels than diabetic groups which were not supplemented with zinc (groups 7 and 8). The report to the effect that zinc supplementation restored the antioxidant activity which was inhibited in rabbits having diabetes induced by streptozotocin (24) is noteworthy in terms of the support it gives to our finding that zinc supplementation increased the GSH values which had been low in the diabetic group as a result of induced diabetes.

A majority of the studies exploring the relation between exercise, lipid peroxidation and antioxidant system have pointed out the increased lipid peroxidation and/or insufficient antioxidant activation in exercise $(9,16)$. Decreased GPx values we obtained after swimming exercise in group 4 in particular are congruous with the results of researchers who reported an inhibition in antioxidant activity as a result of acute exercise. The finding of lowered GPx values in group 7 (diabetic swimming) and group 8 (diabetes) demonstrates that both acute exercise and diabetes can individually inhibit antioxidant activity. It can be claimed that the mechanism of how the antioxidant activity is affected in diabetes has been increasingly attracting attention (19). A host of reports have been published to show the inhibition in GPx activity in diabetic patients and to recommend further research into this inhibition for the benefit of treatment in diabetes $(31,32,33)$. The decreased GPx values we found in groups 7 and 8 are in harmony with the reports of researchers who argued that GPx parameter was inhibited in diabetes. In our study we found the highest GPx values in the zinc-supplemented swimming group (group 5). The report arguing that zinc supplementation inhibited (27) the lipid peroxidation that had increased as a result of physical exercise (15) is noteworthy with regard to the relation between zinc and antioxidant system. The most recent data indicate that zinc can assume important roles in the antioxidant mechanism (25). The fact that we obtained the highest GPx values in the zinc-supplemented swimming group (group 5) demonstrates that lipid peroxidation that increases with exercise can be inhibited by zinc supplementation. Elevated GPx values we found in group 2 (zinc-supplemented control) is proof that a diet rich in zinc, per se, can result in the activation of the antioxidant system. High GPx values we obtained in the zincsupplemented diabetic swimming group are highly impressive. That is because many researchers have noted that GPx activity is suppressed in diabetic patients $(31,32,33)$. A total of 56 adult patients with type 2 diabetes mellitus were supplemented with $30 \mathrm{mg}$ zinc gluconate daily for 6 months to examine the effect of zinc on the antioxidant system (34). Consequently, 6-month zinc supplementation to diabetic patients was found to lead to an increase in plasma zinc levels, decrease in lipid peroxidation, and 
rise in antioxidant activity (34). Results of the concerned study not only demonstrate that zinc supplementation inhibits free radicals that are elevated in diabetes, but also is a strong endorsement of the high GPx values we found in group 6. It is to be emphasized that while Roussel et al (34) reported an increase in antioxidant activity with 6-month zinc supplementation, we obtained similar results in our study with 4 -week zinc supplementation. This indicates that zinc can be effective in the antioxidant system in a relatively short time.

Zinc partakes in the structure of SOD and thus inhibits the free radical chain reaction $(20,25)$. As a consequence of these functions, zinc makes membranes stronger against oxidative stress (35). Considering that zinc takes part in the structure of SOD, high SOD levels found in the zinc-supplemented swimming group (group 5) can be interpreted as an expected result. As Shaheen and el-Fettah (23) reported, zinc-deficient diet alone led to a significant suppression in SOD levels and an increase in free radical formation in rat tissues. Cao and Chen (27) demonstrated in their study that zinc deficiency inhibited SOD levels in mice and caused lipid peroxidation, whereas zinc supplementation increased SOD parameter and prevented lipid peroxidation. The latter is a striking example of the relationship between zinc and SOD. It complies with our result of elevated SOD levels we obtained in group 5. Should the interpretations of reports arguing that zinc-deficient diet per se could inhibit SOD levels (23) and that zinc supplementation brought about an increase in SOD parameter (27) be combined, high SOD levels we obtained in the zinc-supplemented control group (group 2) are consistent with literature data. Elevated SOD levels we found in the zinc-supplemented diabetic swimming group (group 6) constitute a quite important result. One of the remarkable findings of studies involving diabetic patients is the significant impairment of SOD activity (36). The result we obtained in group 6 indicates that SOD activity that is curbed in diabetes is restored by zinc supplementation. Likewise, we found the lowest SOD values in the diabetic group not supplemented with zinc (groups 7 and 8). The increase brought about by zinc in SOD values, which were reduced in diabetes, indicates that zinc supplementation alongside treatment may be significant in diabetes. Likewise, a study in the same vein reported a significant increase in SOD activity of diabetic patients supplemented with zinc (34). Both facts demonstrate the importance of zinc supplementation in diabetes and support the high SOD level we found in the diabetic swimming group supplemented with zinc (group 6).

It was reported that diabetics experienced significant functional impairments in their muscle contractility (37). Impairments of calcium secretion in diabetes lead to dysfunctions in muscle contractions (38), which in turn, caused faster onset of muscle exhaustion. As a matter of fact, the findings of increased lactate levels found after exercise in diabetes (39) or significant increases in lactate levels resulting from high-intensity exercise in diabetics (40) prove that muscle exhaustion occurs earlier in diabetes-exercise correlation. High lactate levels which we identified in the diabetic swimming group are congruous with the reports cited above.

Elevated levels of lactate found in group 4 can be considered an expected result following acute and heavy exercise. What needs emphasis here is that lactate values were lower in group 4 compared to the diabetic swimming group (group 7). This result demonstrates that physical activity conducted under the same conditions and with the same intensity brought about a further increase in diabetics and led to earlier onset of muscle exhaustion. Lactate values of the zinc-supplemented diabetic swimming group (group 6) were significantly lower than in group 4 (swimming control) and group 7 (diabetic swimming). This phenomenon shows us that zinc supplementation can reduce lactate production and delay exhaustion in rats with induced diabetes. Thus, the lactate parameter in the zinc-supplemented swimming group (group 5) was found to be lower than that in group 4 (swimming control), group 6 (zinc-supplemented diabetic swimming) and group 7 (diabetic swimming). This finding suggests that zinc supplementation can delay muscle exhaustion in physical activity. Similarly, a comparison of gastrocnemius muscles taken from rats fed on a zinc-supplemented diet and those of the controls revealed that the former got exhausted later than the latter (41). In another study, where 12 professional football players were subjected to maximum exercise using an ergometer, it was shown that subjects generally had lower serum zinc levels after exercise and that those with low serum zinc levels had high plasma lactate levels (42). This interesting piece of information may trigger research into the effects of zinc, an important trace element, on physiologic performance. An overall evaluation of the data above suggests that zinc can have highly significant effects on muscle functions and can therefore delay muscle exhaustion. Accordingly, we have already shown in our previous study that zinc supplementation to rats subjected to swimming exercise has a protective effect on liver glycogen (43).

It is known that there is a biochemical bond between zinc and insulin (24). Besides, zinc plays a role in the regulation of insulin's effect (2). Body zinc status in diabetic humans and animals is under serious risk due to its high rate of discharge through urine (2, 24). Irreversible loss of zinc causes a decrease in insulin activity, and the resultant development of diabetes and its complications $(3,44)$. Since urinary zinc loss is well-known, we did not determine zinc levels in urine.

Reduced zinc levels we found in diabetic swimming (group 7) and diabetes (group 8) groups result from diabetes and support the above cited studies reporting lowered zinc levels in diabetes. Swimming control group (group 4) had serum zinc levels higher than groups 7 (diabetic swimming) and 8 (diabetes), but lower than all other groups. This finding we obtained in group 4 indicates that acute swimming exercise significantly suppresses serum zinc levels. It was argued that daily and regular exercise could be responsible for the impairment in zinc metabolism and that impairments in zinc metabolism as well as zinc loss could lead to muscle exhaustion and weakness (45). Bordin et al (46) demonstrated that physical exercise led to re-distribution of zinc among body stores, blood and tissues, and the increased metabolism caused zinc deficiency. Lower serum zinc levels that were found in group 4 subjected to acute swimming exercise, are consistent with literature data. The decrease in serum zinc levels following exercise may arise from two distinct events. The first may be associated with modified distribution of zinc from extracellular tis- 
sues to the liver, and the second with high urinary discharge (47). Serum zinc levels in zinc-supplemented diabetic control (group 3) and zinc-supplemented diabetic swimming (group 6) groups were higher than in groups 4 (swimming control), 7 (diabetic swimming) and 8 (diabetes), but lower than all the remaining groups. This particular finding that we obtained in groups 3 and 6 is quite important, since the increase in urinary discharge of zinc in diabetes causes a serious zinc deficiency $(2,24)$ while irreversible loss of zinc brings about diabetic complications $(3,44)$. Thus, the fact that zinc levels which are known to decrease as a result of both. Acute exercise $(45,46)$ and diabetes $(3,44)$ were found to show a marked increase in zinc-supplemented diabetic groups (groups 3 and 6), relative to groups 4, 7 and 8 , indicates the importance of zinc supplementation in association with exercise and diabetes. Several researchers showed that serum zinc values decreased after exercise $(45,46,47)$. Elevated levels of zinc we found in zinc-supplemented swimming group (group 5) point out the significance of zinc supplementation in association with exercise. Bordin et al (46) who demonstrated that physical exercise caused re-distribution of zinc among body stores, blood and tissues and that increased metabolism led to zinc deficiency concluded that these indicators entailed zinc supplementation. Reports of the cited researchers point out the importance of zinc supplementation in association with exercise.

Results obtained from the study indicate that the increase in free radical formation, suppression of antioxidant activity and muscle exhaustion that occur in diabetes and as a result of acute exercise can be prevented by zinc supplementation. Zinc supplementation may contribute to health and performance in diabetes and in association with acute exercise.

\section{References}

1. Laakso M. Cardiovascular disease in type 2 diabetes: challenge for treatment and prevention. J Intern Med 2001; 249: 225-235.

2. Islam MS, Loots du T. Diabetes, metallothionein, and zinc interactions: a review. Biofactors 2007; 29: 203-212.

3. Brandao-Neto J, Silva CAB, Rezende AA et al. Zinc pharmacokinetics in insülin-dependent diabetes mellitus patients after oral zinc tolerance test. Nutr Res 2003; 23: 141-150.

4. Saper RB, Rash R. Zinc: an essential micronutrient. Am Fam Physician 2009; 79: 768-772.

5. Stehbens WE. Oxidative stress, toxic hepatitis, and antiaxidants with particular emphasis in zinc. Exp Mol Pathol 2003; 75: 265-276.

6. Paffenbarger RS, Kampert JB, Lee IM et al. Changes in physical activity and other lifeway patterns influencing longevity. Med Sci Sports Exerc 1994; 26: 857-865.

7. Kim JD, Yu BP, McCarter RJM et al. Exercise and diet modulate cardiac lipid peroxidation and antioxidant defenses. Free Radic Biol Med 1996; 20: 83-88.

8. Powers SK, Hamilton K. Antioxidants and exercise. Clin Sports Med 1999; 18: 525-536.

9. Laaksonen DE, Atalay M, Niskane L, et al. Increased resting and exercise-induced oxidative stress in young IDDM men. Diabetes Care 1996; 19: 569-574.
10. Havel PJ, Uriu-Hare JY, Liu T, et al. Marked and rapid decreases of circulating leptin in streptozotocin diabetic rats: reversal by insulin. Am J Physiol 1998; 274: 1482-1491.

11. Draper HH, Hadley M. Malondialdehyde determination as index of lipid peroxidation. Methods Enzymol 1990; 186: 421-431.

12. Atroshi F, Sankari S, Osterberg S et al. Variation of erythrocyte glutathione peroxidase activity in Finn sheep. Res Vet Sci 1981;31:267-271.

13. Rahimi R, Nikfar S, Larijani B et al. A review on the role of antioxidants in the management of diabetes and its complications. Biomed Pharmacother 2005; 59: 365-373.

14. Kuroki T, Isshiki K, King GL. Oxidative stress: the lead or supporting actor in the pathogenesis of diabetic complications. J Am Soc Nephrol 2003; 4: 216-220.

15. Kanter M. Free radicals, exercise and antioxidant supplementation. Proc Nutr Soc 1998; 57: 9-13.

16. Child RB, Wilkinson DM, Fallowfield JL et al. Elevated serum antioxidant capacity and plasma malondialdehyde concentration in response to a simulated half-marathon run. Med Sci Sports Exerc 1998; 30: 1603-1607.

17. Jenkins RR. Exercise, oxidative stress, and antioxidants: a review. Int J Sport Nutr 1993; 3: 356-375.

18. Vincent AM, Russell JW, Low $P$ et al. Oxidative stress in the pathogenesis of diabetic neuropathy. Endocr Rev 2004; 25: 612-628.

19. Atalay M, Laaksonen DE. Diabetes oxidatife stress and physical exercise. J Sports Sci Med 2002; 1: 1-14.

20. Powell SR. The antioxidant properties of zinc. J Nutr 2000; 130 : $1447-1454$.

21. Oteiza PI, Olin KL, Fraga CG et al. Oxidant defense systems in testes from zinc-deficient rats. Proc Soc Exp Biol Med 1996; 213: 85-91.

22. Oteiza PI, Adonaylo VN, Keen CL. Cadmium-induced testes oxidative damage in rats can be influenced by dietary zinc intake. Toxicology 1999; 137: 13-22.

23. Shaheen AA, el-Fattah AA. Effect of dietary zinc on lipid peroxidation, glutathione, protein thiols levels and superoxide dismutase activity in rat tissues. Int J Biochem Cell Biol 1995; 27: 89-95.

24. Duzguner V, Kaya S. Effect of zinc on the lipid peroxidation and the antioxidant defense systems of the alloxan-induced diabetic rabbits. Free Radic Biol Med 2007; 42: 1481-1486.

25. Prasad AS, Bao B, Beck FW et al. Antioxidant effect of zinc in humans. Free Radic Biol Med 2004; 37: 1182-1190.

26. Cordova A, Alvarez-Mon M. Behaviour of zinc in physical exercise: a special reference to immunity and fatigue. Neurosci Biobehav Rev 1995; 19: 439-445.

27. Cao GH, Chen JD. Effects of dietary zinc on free radical generation, lipd peroxidation, and superoxide dismutase in trained mice. Arch Biochem Biophys 1991; 291: 147 - 153.

28. Jana K, Samanta PK, Manna I et al. Protective effect of sodium selenite and zinc sulfate on intensive swimming-induced testicular gamatogenic and steroidogenic disorders in mature male rats. Appl Physiol Nutr Metab 2008; 33: 903-914.

29. Maritim AC, Sanders RA, Watkins JB. Diabetes, oxidative stress, and antioxidants: a review. J Biochem Mol Toxicol 2003; 17: 24-38.

30. Evans JL, Goldfine ID, Maddux BA et al. Are oxidative stres-activated signaling pathways mediators of insülin resistance and-cell dysfunction. Diabetes 2003; 52: 1-8. 
31. Thornalley PJ, McLellan AC, Lo TW et al. Negative association between erythrocyte reduced glutathione concentration and diabetic complications. Clin Sci 1996; 91: 575-582.

32. Muruganandam A, Drouillard C, Thibert RJ et al. Glutathione metabolic enzyme activities in diabetic platelets as a function of glycemic control. Thromb Res 1992; 67: 385-397.

33. Osterode W, Holler C, Ulberth F. Nutritional antioxidants, red cell membrane fluidity and blood viscosity in type 1 (insulin dependent) diabetes mellitus. Diabet Med 1996; 13: 1044-1050.

34. Roussel AM, Kerkeni A, Zouari $\mathbf{N}$ et al. Antioxidant effects of zinc supplementation in Tunisians with type 2 diabetes mellitus. J Am Coll Nutr 2003; 22: 316-321.

35. Hennig B, Toborek M, McClain CJ. Antiatherogenic properties of zinc: implications in endothelial cell metabolism. Nutrition 1996; 12: 711-717.

36. Kawamura N, Ookawara T, Suzuki $\mathrm{K}$ et al. Increased glycated $\mathrm{Cu}$, Zn-superoxide dismutase levels in erythrocytes of patients with insulindependent diabetus mellitus. J Clin Endocrinol Metab 1992; 74: 1352-1354.

37. Katta A, Preston DL, Karkala SK et al. Diabetes alters contractioninduced mitogen activated protein kinase activation in the rat soleus and plantaris. Exp Diabetes Res 2008; 2008: 738101.

38. Reuter H, Grönke S, Adam C et al. Sarcoplasmic Ca2+ release is prolonged in nonfailing myocardium of diabetic patients. Mol Cell Biochem 2008; 308: 141-149.

39. Guelfi KJ, Jones TW, Fournier PA. The decline in blood glucose levels is less with intermittent high-intensity compared with moderate exercise in individuals with type 1 diabetes. Diabetes Care 2005; 28: 1289-1294.
40. Bussau VA, Ferreira LD, Jones TW et al. A 10-s sprint performed prior to moderate-intensity exercise prevents early post-exercise fall in glycaemia in individuals with type 1 diabetes. Diabetologia 2007; 50: $1815-1818$.

41. Richardson JH, Drake PD. The effects of zinc on fatigue of striated muscle. J Sports Med 1979; 19: 133-134.

42. Khaled S, Brun JF, Bardet $L$ et al. Physiological importance of zinc in muscular activity. Sci Sports 1997; 12: 179-191.

43. Bicer M, Gunay M, Akil M et al. Effect of Long-Term Intraperitoneal Zinc Administration on Liver Glycogen Levels in Diabetic Rats Subjected to Acute Forced Swimming. Biol Trace Elem Res 2010; DOI 10.1007/s2011-010-8658-5.

44. DiSilvestro RA. Zinc in relation to diabetes and oxidative disease. J Nutr 2000; 130: 1509-1511.

45. Cordova A, Navas FJ. Effect of training on zinc metabolism: changes in serum and sweat zinc concentrations in sportsmen. Ann Nutr Metab 1998; 42: 274-282.

46. Bordin D, Sartorelli L, Bonanni G et al. High intensity physical exercise induced effects on plasma levels of copper and zinc. Biol Trace Elem Res 1993; 36: 129-134.

47. Campbell WW, Anderson RA. Effects of aerobic exercise and training on the trace minerals chromium, zinc and copper. Sports Med 1987; 4: 9-18.

Received November 16, 2011. Accepted November 26, 2011. 